\title{
ANALYTICAL MIMO-OFDM SYSTEM REPRESENTATION BASED ON FRACTIONAL FOURIER TRANSFORM
}

\author{
Hanadi Mohammed Abass ${ }^{1}$, Mohammed $\mathrm{Ali}^{2}$, Sha Xuejun ${ }^{3}$
}

\begin{abstract}
The demand for wireless systems has been growing rapidly over the recent years due to improved reliability, high data rates, seamless connectivity and low deployment costs. MIMO systems are the most efficient leading innovation of wireless systems for maximum capacity and improved quality and coverage. Multiple Input Multiple Output (MIMO) in combination with Orthogonal Frequency Division Multiplexing (OFDM) can provide spectrally efficient and ISI free communication. The Fractional Fourier transform (FrFT), as a generalization of the classical Fourier Transform, was introduced many years ago in mathematics literature. In this paper selecting the optimal order of the fractional Fourier transform, the modulated signals can match the time-varying channel characteristics, which results in a mitigation of the inter carrier interference. Furthermore, an algorithm is presented for selecting the optimal order of fractional Fourier transform, and the impact of system parameters on the optimal order is analyzed. Experimental results yields good results over conventional state of art methods.
\end{abstract}

Keywords-Fractional Fourier transform, MIMOOFDM, inter carrier interference

\section{Introduction}

Multiple Input Multiple Output (MIMO) systems have been recently emerged as a key technology in wireless communication systems for increasing both data rates and performance[1,2]. MIMO is an antenna

Hanadi Mohammed Abass ${ }^{1}$

Engineering college / Alzaeim Alazhari University

Sudan.

Mohammed $\mathrm{Ali}^{2}$

Engineering college / Khartoum University .

Sudan.

Sha Xuejun ${ }^{3}$

Communication Research Center / Harbin Institute of Technology China.

technology that used both in transmitter and receiver equipment for wireless radio communication. MIMO uses multiple antennas to send multiple parallel signals for transmission[3]. Wireless communication technology has shown that when multiple parallel signals for transmission[3].

Wireless communication technology has shown that when multiple antennas at both transmitter and receiver are employed it provides the possibility of higher data rates compared to single antenna systems. MIMO exploits the space dimension to improve wireless system capacity, range and reliability. In the never ending search for increased capacity in a wireless communication channel it has been shown that by using MIMO OFDM systems it is possible to increase that capacity substantially[4-6]. Especially in broadband application where Inter-symbol Interference is a critical factor. Equalizers are employed to reduce such interference. MIMO system transmits different signals from each transmit element so that the receiving antenna array receives a superposition of all the transmitted signals. MIMO has eminent features which offer significant increment in data throughput and link range without additional bandwidth and increase transmit power[10].

Channel estimation is an important technique especially in mobile wireless network systems. The knowledge of the impulse response of mobile wireless propagation channels in the estimator is an aid in acquiring important information for testing, designing or planning wireless communication systems. Channel estimation is based on the training sequence of bits and which is unique for a certain transmitter and which is repeated in every transmitted burst. The channel estimator gives the knowledge on the channel impulse response (CIR) to the detector and it estimates separately the CIR for each burst by exploiting transmitted bits and corresponding received bits. Signal detectors must have knowledge concerning the channel impulse response (CIR) of the radio link with known transmitted sequences, which can be done by a separate channel estimator.

The use of MIMO technology in combination with OFDM therefore seems an attractive solution for future wireless communication. This paper implements channel estimation in MIMOOFDM system for Spatial Multiplexing technique with the aid of pilot symbols to estimate the channel. Least Square (LS) is used to get the initial estimation, 
whereas to define all of channel characteristics, we use the channel condition in the past in all subcarriers with no pilot symbols. The performance is measured by implementing channel estimation in applicable proposed system design and pilot type.

\section{Related Content}

\section{A. Basic OFDM System}

The OFDM block diagram is illustrated as follows in figure 1 . The input random signal data rate streams (high) are converted into data rate streams (low). The important aspect in the OFDM block diagram is the modulation technique which modulat-

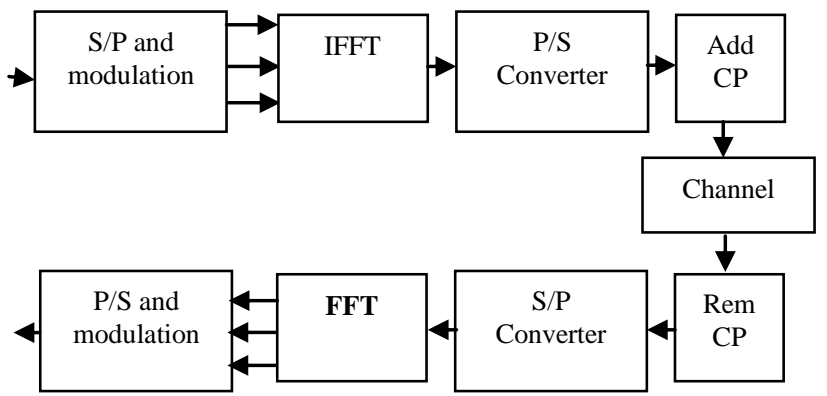

Figure 1: Block diagram of Basic OFDM system

-es the low data rate streams in parallel way and this parallel stream given input to the IFFT block which transforms the frequency data to time data before it reaches the channel. Adding the cyclic prefix acts as

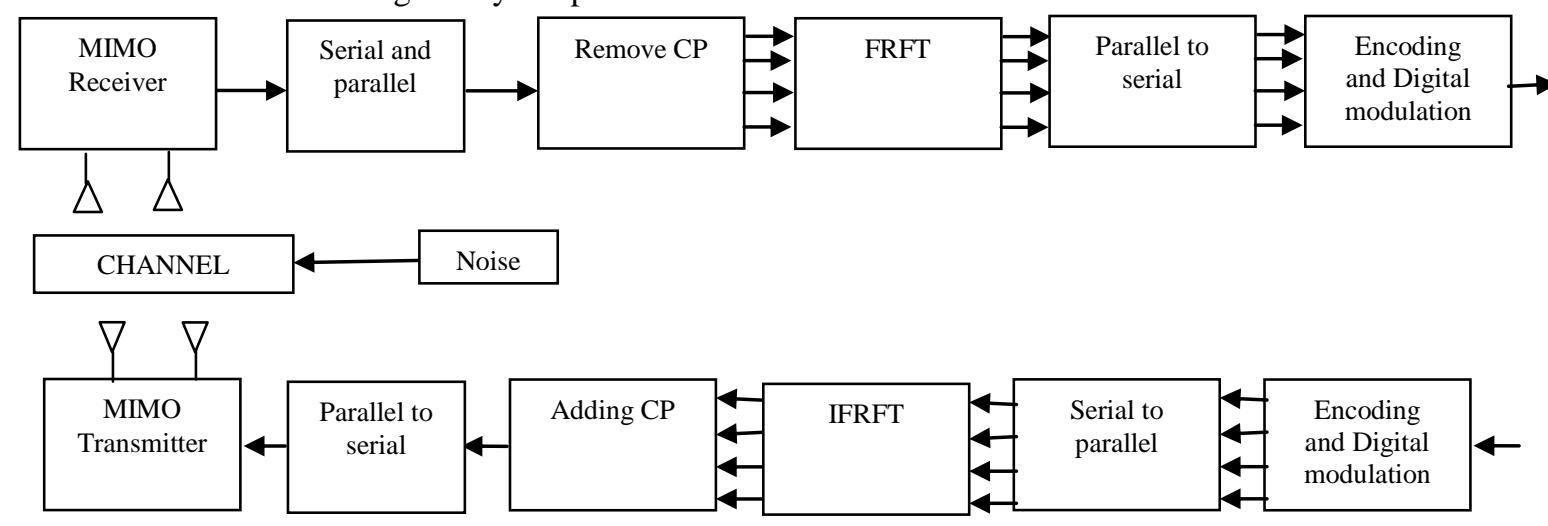

Figure 2: Block diagram of MIMO OFDM system

\section{Proposed method}

\section{A. FrFT-based MIMO-OFDM system}

A MIMO- OFDM system with $M_{t}$ transmit antennas and $M_{y}$ receive antennas for FRFT as shown in figure 2. In transmitter section the usage of space time coding is witnessed to map the information bearing sequence in transmit antennas in reliable way. The data sequence is initially converted to the guard interval and the reverse of transmission is accomplished at receiver end.

\section{B. MIMO OFDM system}

The below block diagram fig(2) represents the MIMO-OFDM system which comprises of transmitters and receivers in multiple way. The input data (digital) is generated by binary source generator as shown in below figure and the binary data is modulated with modulation approach such as BPSK, QPSK and QAM with several different constellations. The serial to parallel performs the task to convert the serial data to the parallel mode in $\mathrm{N}$ various sub streams. Then these various sub streams are modulated through the IFFT modulation block.

The IFFT block in the block diagram in design to transform the frequency to time domain for obtaining the delay related issues at the channel and then guard interval named CP is inserted to tackle the issues like ICI/ISI. The OFDM symbols are initialized in the time domain which has specified length before giving it to the channel then the operation is performed in the inverse direction to remove all the operations which are performed and gets the output as OFDM signal in MIMO format. The received data are first converted in serial to parallel form data from time domain symbol in frequency domain and again covert it parallel to serial form. When we have to demodulate and decode the data, we get estimated output. The MIMO-OFDM system has multiple input and multiple output. 
fractional?" First we see a transformation T, we can describe the transformation as following:

$$
T\{f(x)\}=F(u)
$$

where $\mathrm{f}$ and $\mathrm{F}$ are two functions with variables $\mathrm{x}$ and $\mathrm{u}$ respectively. As seen, we can say that $\mathrm{F}$ is a $\mathrm{T}$ transform off. Now, another new transform can be defined as below:

$$
T^{\alpha}\{f(x)\}=F_{\alpha}(u)
$$

We call $T^{\alpha}$ here the " $\alpha$-order fractional T transform" and the parameter $\alpha$ is called the "fractional order". This kind of transform is called "fractional transform". Which satisfy following Boundary conditions :

$$
\begin{aligned}
& T^{0}\{f(x)\}=f(u) \\
& T^{1}\{f(x)\}=F(u)
\end{aligned}
$$

\section{B. Encoding of STBC and SFBC}

In a given Alamouti code block, two symbols of s1 and s2 are encoded using the following orthogonal

$\operatorname{matrix} \mathbf{A}=\left[\begin{array}{cc}\mathrm{S}_{1} & \mathrm{~S}_{2} \\ -\mathrm{S}_{2}^{*} & \mathrm{~S}_{1}^{*}\end{array}\right]$

The encoding matrix defines the transmission format with the row index indicating the antenna number and the column index indicating the OFDM symbol index (sub-carrier index) for STBC (for SFBC).

\section{Subcarrier mapping for STBC}
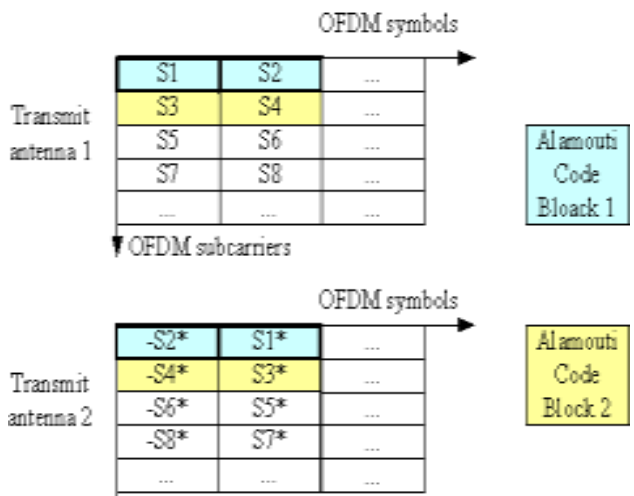

OFDM subcamers

Figure 3: An example of STBC encoding

For STBC, a pair of symbols, s1 and s2, are encoded into four variants, $\mathrm{s} 1, \mathrm{~s} 2,-\mathrm{s} 2^{*}$, and $\mathrm{s} 1^{*}$. As illustrated in Figure $1, \mathrm{~s} 1$ is transmitted over a certain sub-carrier from antenna one, and $-\mathrm{s} 2^{*}$ is over the same subcarrier from antenna two.
During the next OFDM symbol, s2 and $\mathrm{s} 1^{*}$ are mapped onto the same sub-carrier from the two antennas. That is, each symbol (or its positive/negative conjugate) is transmitted from two antennas and over two OFDM symbols.

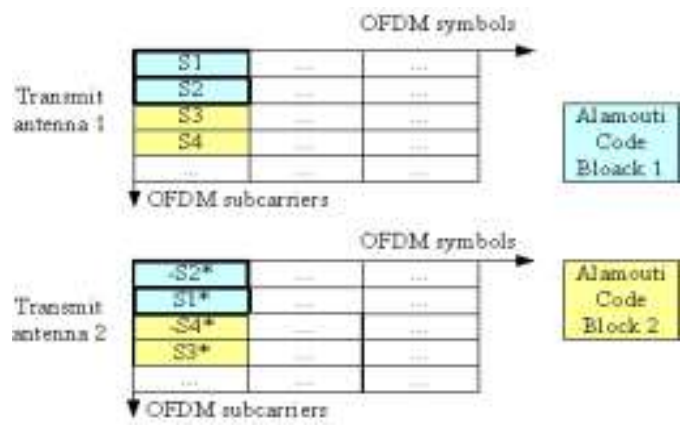

Figure 4: An example of SFBC encoding

\section{Subcarrier mapping for SFBC}

As depicted in Figure 2, SFBC also encodes a pair of symbols, s1 and s2 into four variants, s1, s2, $-\mathrm{s} 22^{*}$, and $\mathrm{s} 1^{*}$, and transmits $\mathrm{s} 1$ and $-\mathrm{s} 2 *$ over a certain sub-carrier from the two antennas. However, the other two variants, s2 and s1*, are transmitted from the subsequent contiguous or discontinuous sub-carriers. That is, each symbol (or its positive/negative conjugate) is transmitted from two antennas and over two sub-carriers (rather than over two OFDM symbols in STBC).

\section{System Transmission model}

We take here a baseline configuration of MIMO with two transmit antennas and two receive antennas as an example to show the transmission model. The same principle is feasible for other MIMO configurations with two transmit antennas and multiple receive antennas. Assuming $\mathbf{H}^{1}=\left(\begin{array}{ll}h_{11}^{1} & h_{12}^{1} \\ h_{21}^{1} & h_{22}^{1}\end{array}\right)$ and $\mathbf{H}^{2}=\left(\begin{array}{ll}h_{11}^{2} & h_{12}^{2} \\ h_{21}^{2} & h_{22}^{2}\end{array}\right)$ be channel matrix of receive/transmit antenna pairs during the $1^{\text {st }}$ and $2^{\text {nd }}$ symbol/subcarrier intervals of Alamouti code, respectively. In the equations, $h_{m n}^{k}$ denotes the channel fading coefficient between the $m$ th receive antenna to the $n$th transmit one during the $k$ th symbol/subcarrier intervals. Then, the MIMO channel is quasi-static if $\mathbf{H}^{1} \approx \mathbf{H}^{2}$, otherwise the MIMO channel is selective.

\section{A. Quasi-static flat fading channel}

In the design of Alamouti code, quasi-static flat fading channels are assumed, which makes it possible to use simple decoder to achieve full spatial diversity. In this case, the received signals can be expressed as 
$Y^{1}=\left[\begin{array}{l}y_{1}^{1} \\ y_{2}^{1}\end{array}\right]=\mathbf{H}^{1}\left[\begin{array}{c}s_{1} \\ -s_{2}^{*}\end{array}\right]+\left[\begin{array}{l}n_{1}^{1} \\ n_{2}^{1}\end{array}\right]$

and :

$$
Y^{2}=\left[\begin{array}{l}
y_{1}^{2} \\
y_{2}^{2}
\end{array}\right] \approx \mathbf{H}^{1}\left[\begin{array}{l}
s_{2} \\
s_{1}^{*}
\end{array}\right]+\left[\begin{array}{l}
n_{1}^{2} \\
n_{2}^{2}
\end{array}\right]
$$

where $s_{i}(i=1,2)$ is the transmitted symbols, $y_{i}^{j}$ $(i, j=1,2)$ is the received signal during the $j$ th symbol/subcarrier interval over $i$ th antenna; $h_{i j}^{1}(\mathbf{i}=1,2$ and $j=1, \ldots, m)$ is the fading coefficient during the $1^{\text {st }}$ symbol/subcarrier interval; and $n_{i}^{j}$ is the additive Gaussian noise with zero mean - and variance - $N_{0}$ during the $j$ th symbol interval over the $i$ th antenna.

\section{B. Selective fading channel}

In a selective fading channel where the quasi-static assumption doesn't hold, the receive signals will be expressed as

$$
Y^{1}=\left[\begin{array}{l}
y_{1}^{1} \\
y_{2}^{1}
\end{array}\right]=\mathbf{H}^{1}\left[\begin{array}{c}
s_{1} \\
-s_{2}^{*}
\end{array}\right]+\left[\begin{array}{l}
n_{1}^{1} \\
n_{2}^{1}
\end{array}\right]
$$

and :

$$
Y^{2}=\left[\begin{array}{l}
y_{1}^{2} \\
y_{2}^{2}
\end{array}\right]=\mathbf{H}^{2}\left[\begin{array}{l}
s_{2} \\
s_{1}^{*}
\end{array}\right]+\left[\begin{array}{l}
n_{1}^{2} \\
n_{2}^{2}
\end{array}\right](10)
$$

\section{Decoding of $S T B C / S F B C$}

Maximum likelihood decoder using only linear processing at the receiver is used for decoding Alamouti code. In order to analyze the impact of channel selectivity in an Alamouti code on its performance, MMSE decoder is also used for a reference to reduce inter-symbol interference (ISI) due to channel selectivity.

\section{Results}

In this proposed method, No. of symbols 1024 are used as data signal and 16 sub carries are used to transmit these symbols. In this technique, Rayleigh channel is used to transmit the symbols.

\section{A. Analysis of figure(4)}

As shown in figure1, the graph is represented between BER and SNR (signal to noise ratio). As the graph indicates, the performance of BER reduces automatically when SNR value increases. Because, as the BER decreases the data rate increases.

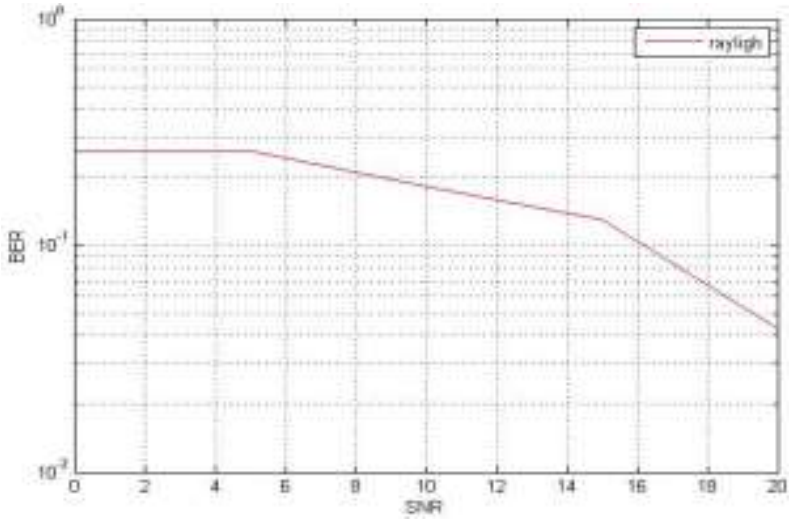

Figure 4 performance of BER in Rayleigh channel

\section{B. Analysis of figure(5)}

The performance of carrier to interference ratio, as the CIR reduces the system capacity increases automatically. As the CIR reduces the performances of data rate increases and speed increases and the interference decreases.

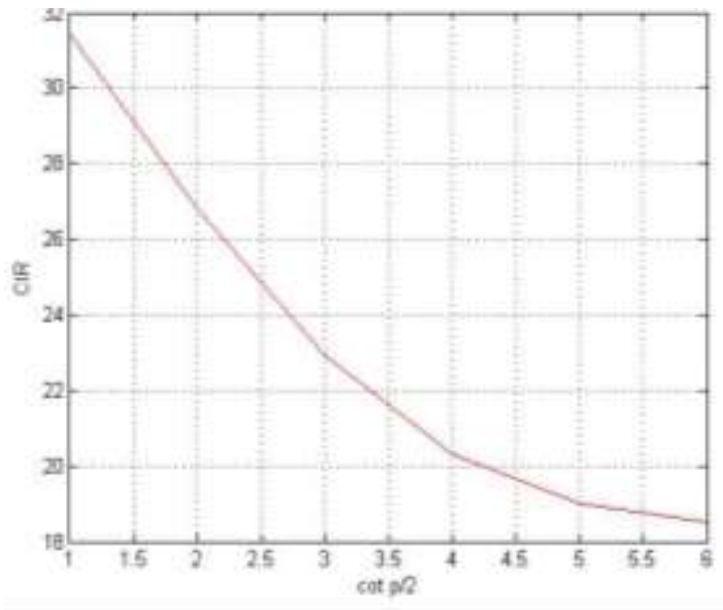

Figure 5 Carrier to interference ratio

Figure 5 indicates $\mathrm{X}$ axis as system performance and $\mathrm{Y}$ axis shows carrier to interference ratio

\section{Analysis of figure(6)}

The above graph indicates $\mathrm{X}$-axis as Bit error rate and $\mathrm{Y}$ axis as signal noise to interference ratio. As SNIR decreases system performance increases and the interference of the signal decreases automatically.

\section{Analysis of figure(7)}

The above graph shows the performance of channel estimation. The $\mathrm{X}$-axis shows signal to noise ratio and $\mathrm{Y}$-axis shows normalized mean square error. In the above box RayH11 indicates 1st transmitter is connected to 1 st receiver as so on. The performance of the signal should be increased linearly. 
Proc. of the Fifth Intl. Conf. Advances in Computing, Communication and Information Technology- CCIT 2017 Copyright (C) Institute of Research Engineers and Doctors, USA .All rights reserved.

ISBN: 978-1-63248-131-3 doi: 10.15224/ 978-1-63248-131-3-30

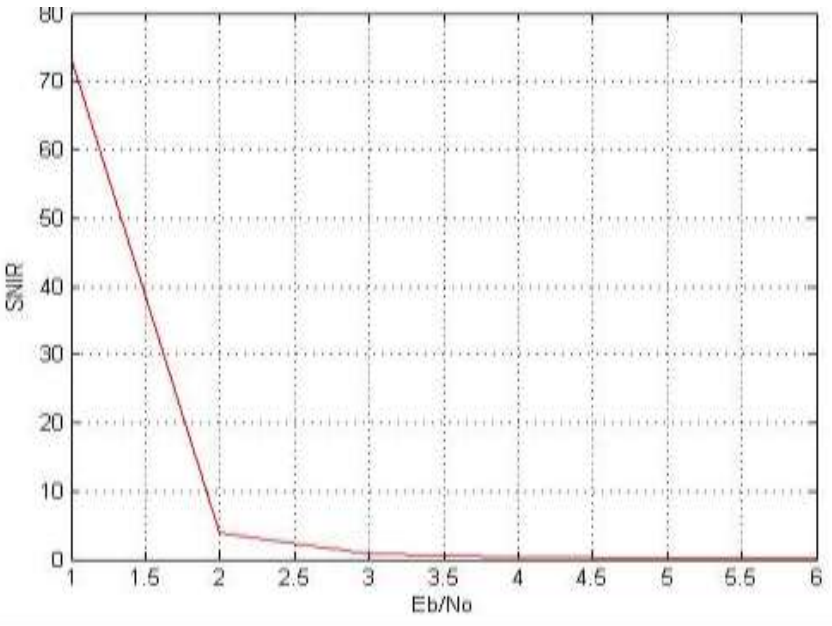

Figure 6 signal to noise interference ratio

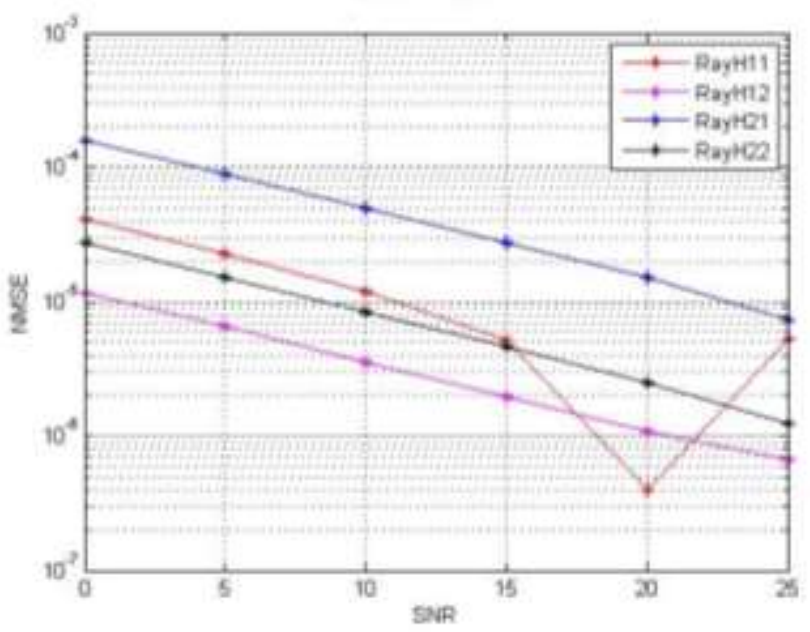

Figure 7 Channel estimation

\section{Conclusion}

MIMO systems are efficient leading innovation of wireless systems for maximum capacity, improved quality and coverage. Multiple Input Multiple Output (MIMO) combines with OFDM provides spectrally efficient and ISI free communication. The Fractional Fourier transform (FrFT), as a generalization of the classical Fourier Transform, was introduced many years ago in mathematics literature. In thispaper selecting the optimal order of the fractional Fourier transform, the modulated signals can match the time-varying channel characteristics, which results in a mitigation of the inter carrier interference.

\section{References}

1 Foschini G J, Gans M J. On limits of wireless communication in a fading environment when using multiple antennas. Wirel Pers Commun, 1998, 6(3): $311-335$

2 Jankiraman M. Space-Time Codes and MIMO Systems. BostonLondon: Artech House Publishers, 2004. 1-12

3 Wang Z, Giannakis G B. Wireless multicarrier communications: where Fourier meets Shannon. IEEE Signal Process Mag, 2000, 17(3): 29—48
4 Stuber G L, Barry J R, McLaughlin S W, et al. Broadband MIMOOFDM Wireless Communications. Proc IEEE, 2004, 92(2): 271-294

5 Stamoulis A, Diggavi S N Al-Dhahir N. Intercarrier Interference in MIMO OFDM. IEEE Trans Signal Process, 2002, 50(10): 2451—2464

6 Song L J, Mohsen K. Effects of time selective multipath fading on OFDM systems for broadband mobile application. IEEE Commun Lett, 1999, 3(12): $332-334$

7 Vahlin A, Holte N. Optimal finite duration pulses for OFDM. IEEE Trans Commun, 1996, 44(1): 10-14

8 Kozek W, Molisch A. Nonorthogonal pulseshapes for multicarrier communications in doubly dispersive channels. IEEE J Sel Area Commun, 1998, 16(8): 1579-1589

9 Strohmer T, Beaver S. Optimal OFDM design for time-frequency dispersive channels. IEEE Trans Commun, 2003, 51(7): 1111-1122

10 Martone M. A multicarrier system based on the fractional Fourier transform for time-frequency selective channels. IEEE Trans Commun, 2001, 49(6): $1011-1020$

About Author (1):

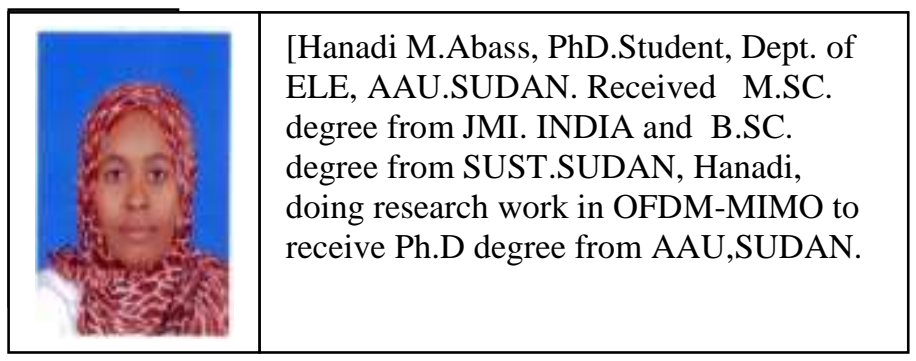

About Author (2):

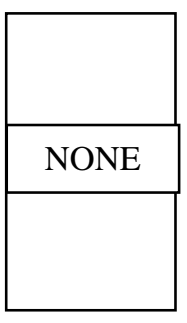

[Mohammed Ali,Assistant Professor in Dept. of ELE,

UOFK. currently supervise number of researchers working in Digital Signal Processing.

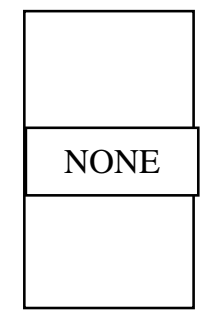

[Sha Xuejun Professor in Communication Research Center, Harbin Institute of Technology, Harbin, Heilongjiang, China, 150001. currently supervise number of researchers working in Digital Signal Processing- MIMO. 